\title{
Measures to Prevent Healtcare Workers from Contracting Severe Acute Respiratory Syndrome During High-Risk Surgical Procedures
}

Published online: 8 January 2004

(C) Springer-Verlag 2004

Severe acute respiratory syndrome (SARS) is a contagious viral disease caused by a novel coronavirus (SARS$\mathrm{CoV}$ ) and transmitted through droplets and close contact [1]. Healthcare workers (HCWs) are particularly at risk when looking after SARS patients. As reported by the World Health Organization [2] more than 20\% (386) of those infected with SARS in Hong Kong were HCWs. Described here are the infection control procedures undertaken at a hospital in Hong Kong when three Caesarean sections were performed on women with active maternal SARS infection.

SARS-CoV infection had been confirmed by reverse transcriptase polymerase chain reaction in all three mothers prior to the time of operation. The operations were performed between 1 April and 5 April 2003. None of the HCWs $(>15)$ involved in the three separate operations developed fever or clinical SARS within 14 days of exposure.

When the operations were performed, the Centers for Disease Control and Prevention (CDC; Atlanta, Ga., USA) had not yet prepared guidelines for the prevention of SARS transmission during Caesarean sections. Since these procedures are associated with the use of suction irrigation and diathermy, it was likely that body fluid and blood would spill and evaporate into the room during the operation. Therefore, the procedures were assumed to be aerosol generating, and previously described precautions to prevent contact and airborne infection were undertaken

S. F. Wong $(-$ K. M. Chow · Y. P. Leung · A. Chiu ·

P. W. Y. Lam - L. C. Ho

Department of Obstetrics and Gynaecology,

Maternal Fetal Medicine Unit,

Princess Margaret Hospital,

Lai Chi Kok, Kowloon, Hong Kong

e-mail: shellwong@hotmail.com

Tel.: +1-852-29901111

Fax: +1-852-29903488

C. C. Shek

Department of Paediatric and Adolescent Medicine,

Princess Margaret Hospital,

Lai Chi Kok, Kowloon, Hong Kong to prevent nosocomial SARS infection [3]. Although the CDC provided guidelines for the prevention and control of SARS infection on 6 May 2003 [4], those guidelines have, to the best of our knowledge, never been tested for the procedure described here. Following are the steps and procedures we undertook to prevent HCWs in our hospital from contracting SARS.

For the three Caesarean sections performed on mothers with SARS, the number of healthcare workers was limited to a minimum, with only those personnel essential to carry out the operation, neonatal resuscitation, and cleanup being involved (i.e., 2 senior obstetricians, 2 senior neonatologists, 1 senior anaesthetist, 1 theatre assistant, a team of 4 senior midwives, and 2 cleansing staff). A nurse supervisor was designated as in-charge and was responsible for monitoring the other $\mathrm{HCWs}$ with regard to the use of personal protective equipment (PPE), cleansing of the room, sterilising the equipment and transferring the patients. She ensured all HCWs had fit-tested their respirators. She was also responsible for testing the portable high-efficiency particulate air filter (HEPA) units (Air-Mate PAPR; USA), checking the batteries, and training other HCWs on the proper use of the HEPA units.

All unnecessary instruments were removed from the operating theatre. Extra laparotomy instruments were prepared to deal with possible intra-operative complications. Two bags of blood were made available in the theatre for the operation. Disposable instruments were used if available. To avoid spillage of blood, drapes with plastic bags on the sides were used.

An operating theatre separate from the main theatre block was designated for this type of surgery in order to minimise contamination of the main theatre. The door of the operating theatre was kept closed during the operation, except when participating personnel entered or left the room. In order to keep the number of entrances and exits to a minimum during the operation, most of the equipment was prepared before the patient arrived. The air circulation was adjusted to 20 exchanges per minute. Negative pressure was created within the operating theatre relative to the adjacent room or hallway; thus, 
recirculation of the room air was avoided. The hospital engineer inspected and checked the air circulation prior to each operation.

The participating HCWs wore appropriate PPE according to the hospital's guidelines prior to the arrival of the patient from the intensive care unit. Every HCW wore a disposable cap, a protective waterproof gown, gloves, N95 respirators, goggles/face shield and waterproof boot cover. In addition, all HCWs working inside the operating theatre wore HEPA units. The HEPA units each had a hood that covered the face and shoulders, and this was connected to a breathing tube on the portable HEPA unit. A non-sterile waterproof protective gown was then put on. After donning this protective gear, the surgeons performed standard scrub then put on sterile waterproof gowns and two pairs of sterile gloves.

HCWs were advised to avoid touching their faces and the PPE on their faces with contaminated gloves. They were also advised to avoid contaminating surfaces around the room. Before leaving the operating theatre, the soiled gowns, gloves and boot covers were removed. An assigned HCW wearing appropriate PPE then wiped the remaining external outfits of the theatre personnel with a diluted sodium hypochlorite solution (a disinfectant, diluted 1 in 49). The remaining outfit was then removed carefully preventing contamination of skin, mucous membranes and clothing. All HCWs washed their hands with chlorhexidine solution, (Hibiscrub; AstraZeneca PLC, UK) and took full-body showers after the operation.

All three patients had been intubated and ventilated prior to the decision for Caesarean section being made. However, in the absence of an effective filtering valve, they were likely to disseminate virus through the endotracheal tube while being transferred from the intensive care unit. To avoid this, a bacterial/viral filter on an exhalation valve was fitted to the endotracheal tube prior to transfer to the operating theatre.

The neonatal resuscitator was placed outside the operating theatre to (i) reduce the number of HCWs inside, (ii) reduce the number of entries to the theatre, and (iii) reduce the exposure of the newborn to the viral load in the environment. Neonatologists wore the PPE described above, including HEPA units with face masks, but their ears were exposed to enable auscultation with a stethoscope during the resuscitation procedure. After birth, thorough suctioning of the newborn's nasopharyngeal tract was performed prior to the first breath. The newborns were wiped dry of blood and amniotic fluid with a sterile cloth before being transferred to the resuscitator.

Routine suction of the mouth and nose was not performed. If suction was required, it was performed using the vacuum suction system on the wall with a sealed system. Suction attached to the resuscitator was avoided, since the gas aspirated would be released directly into the adjacent environment. Bagging with a face mask was kept to a minimum and direct intubation, using a closed suction system, was performed more readily. After stabilisation, the newborns were wrapped in sterile linens and transferred to the isolation nursery using transport incubators. The newborns were kept in the isolation nursery until proven non-contagious. None of the three newborns had any clinical evidence of SARS and paired SARS-CoV antibody titres were not raised.

After the operation, sodium hypochlorite diluted 1:49 was used to clean the floor, the operating table and environmental surfaces as soon as possible. The cleaners all wore appropriate PPE as described above. All waste materials and disposable instruments were treated as highly contagious and were disposed of appropriately according to the hospital's guidelines. Samples collected for pathology and microbiology were placed in two clean plastic bags. If there was evidence of soiling, an extra plastic bag was to be used. Maternal SARS infection was clearly labeled on the laboratory forms.

The optimal PPE for preventing transmission of SARS during such ultra-high-risk procedures has not yet been determined. However, PPE should be used that covers all exposed areas of skin including the arms, torso, eyes, nose and mouth. N95 or higher standard respirators are also necessary. A fit-check should be performed each time a respirator is put on. For better protection of staff working inside operating theatres, we used HEPA units with hoods completely covering the head and neck and portions of the shoulders and torso. Factors to consider when choosing respirators in this setting include availability, impact on mobility, comfort, duration of use and recharge power supply. With the units we used, the HCWs did not encounter any major difficulty, apart from slightly reduced mobility and difficulty in communicating while carrying out the operation. Some problems known to be associated with HEPA units include partially charged batteries, ineffective filters, and contamination of HCWs during disrobing. Proper training prior to use may reduce such problems.

Negative-pressure air circulation helps to reduce the viral load within the operating theatre. The main con-cern with this practice, however, is that by drawing air from the hallway a high flow of unfiltered air is created, which may increase the risk of wound infection. Two of our three patients who underwent surgery developed wound infections, but it is difficult to attribute these infections with certainty to either the effect of the high-dose steroid given and/or the negative-pressure air circulation.

In conclusion, the procedures described above were sufficient to prevent our healthcare workers from contracting SARS while performing these very high-risk operations. Therefore, we advocate the use of such measures or those with higher levels of precaution. We cannot comment on the effectiveness of less stringent measures in minimising SARS transmission. 


\section{References}

1. Peiris JSM, Lai ST, Poon LLM, Guan Y, Yam LYC, Lim W, et al. (2003) Coronavirus as a possible cause of severe acute respiratory syndrome. Lancet 361:1319-1325

2. World Health Organisation (2003) Severe acute respiratory syndrome (SARS)http://www.who.int/csr/sars/
3. TB Infection-Control Guidelines Workgroup (1994) Guidelines for preventing the transmission of Mycobacterium tuberculosis in health-care facilities. MMWR 43:1-132

4. Centers for Disease Control (2003) SARS infection control and exposure management.http://www.cdc.gov/ncidod/sars/ic.htm 